\title{
Business Intelligence: métodos e técnicas de gestão do conhecimento e as tendências para avanços do capital intelectual
}

\author{
Bruna Devens Fraga Doutoranda em Engenharia e Gestão do Conhecimento. Universidade Federal de Santa Catarina (UFSC) - Brasil. brunadefraga@gmail.com \\ Julio Graef Erpen Doutoranda em Engenharia e Gestão do Conhecimento. Universidade Federal de Santa Catarina (UFSC) - Brasil. \\ jgerpen@yahoo.com.br \\ Gregorio Varvakis Doutor em Manufacturing Engineering. Universidade Federal de Santa Catarina (UFSC) - Brasil. gvarvakis@hotmail.com \\ Neri dos Santos Doutor em Ergonomie de I'Ingenierie. Pontifícia Universidade Católica do Paraná (PUCPR) - Brasil. nerisantos@gmail.com
}

\section{RESUMO}

A demanda por bens e serviços nos sistemas produtivos exige a gestão de seus ativos intangíveis. Torna-se imperativa a gestão do conhecimento nas organizações por meio de métodos e técnicas, como instrumento de evolução e aprendizagem organizacional. A pesquisa identificou os métodos e técnicas de Gestão do Conhecimento (GC) para a adoção de uma cultura orientada a dados e correlacionou com Capital Intelectual (humano, estrutural e relacional). Amostragem incluiu 20 empresas de uma associação de classe. As práticas de 'ajuda a semelhante', 'comunidades de prática' e 'benchmarking interno e externo' relacionaram-se ao capital humano e relacional. 'Elaboração de manuais para replicação de práticas' convergiu com o capital humano. 'Visitas técnicas entre as empresas' e 'coaching' com capital relacional. 'Espaços eletrônicos' e 'gestão eletrônica de documentos' indicou dimensões do capital humano e estrutural, relacionados ao conhecimento de competências individuais e organizacionais. $\mathrm{O}$ trabalho recomendou métodos e técnicas essenciais e alinhados às tendências do Business Inteligence (BI).

Palavras-chave: métodos e técnicas de GC; capital intelectual; business intelligence.

\section{Business Intelligence: methods and knowledge management techniques and trends in the advancement of intellectual capital}

\begin{abstract}
Demand for goods and services in production systems requires the management of their intangible assets. Knowledge management in organizations through methods and techniques such as development tool and organizational learning become imperative This article identified the methods and Knowledge Management techniques to adopt a culture based on data and correlated with intellectual capital (human, structural and relational). Sample included 20 companies from a trade association. Practices such as 'help to the like', 'community of practice' and 'internal and external benchmarking' were related to the human and relational capital. 'Manual elaboration for replication practices' converged with human capital. 'Technical visits to companies' and 'coaching' had to do with relational capital. 'Electronic Spaces' and 'electronic document management' indicated dimensions of human and structural capital, related to knowledge of individual and organizational skills. The research recommended essential methods and techniques aligned to trends in Business Intelligence (BI).
\end{abstract}

Keywords: KM practice and tools; intellectual capital; business intelligence. 


\section{Introdução}

Os sistemas produtivos contemporâneos, nas próximas décadas, deverão se adaptar aos mercados globalizados e direcionar seus ativos intangíveis de conhecimentos à aprendizagem organizacional, propriedade intelectual e a pressão crescente da sociedade exigente por serviços e bens oriundos de modelos que preconizem o desenvolvimento sustentável.

As ações de aquisição, armazenamento e compartilhamento de conhecimentos, de modo a aprender as lições do passado e de outros lugares - a superação dos limites impostos pelo tempo e pelo espaço - estão longe de serem novidades. Nos últimos anos, atuações nesse sentido enfatizaram a melhor aplicação do conhecimento e da aprendizagem como uma forma de melhoria do trabalho e o desenvolvimento humanitário.

Segundo a Asian Productivity Organization, desde o início de 1990, o paradigma da competição mudou gradualmente, "da guerra da eficiência para a guerra para a inovação como o campo de batalha" (Asian Productive Organization [APO], 2009, p. 01). E, nesse aspecto, Drucker (2001) afirmava que, na sociedade do conhecimento, se viveriam mudanças de paradigmas, como foco inquestionável de transformação: "o conhecimento como fator-chave de riqueza $e$ prosperidade".

O presente trabalho surgiu do questionamento: Quais são as possibilidades de métodos e técnicas de GC para a adoção de uma cultura orientada a Bussines Inteligence e ao Capital Intelectual dentro de uma organização? Para tal a pesquisa objetivou identificar os métodos e técnicas de GC utilizados em uma Organização Intensiva em Conhecimento (OIC), correlacionar os métodos e técnicas nas dimensões do Capital Intelectual $(\mathrm{Cl})$ e recomendar os métodos e técnicas que estão alinhados às tendências do Business Inteligence.

\section{Desenvolvimento Teórico}

\section{Gestão do Conhecimento: Métodos e Técnicas}

O acesso à informação e a capacidade de colocar o conhecimento para uso produtivo sempre foram a marca registrada de pessoas bem sucedidas, empresas e até mesmo nações. Assim, a consideração de que o conhecimento tem grande valor, tem estado presente ao longo do tempo. Mas, até recentemente, a maioria das pessoas não pensavam em termos de "gestão do conhecimento". Em vez disso, elas consideravam o conhecimento como um bem pessoal, que é a soma de nossas experiências, educação e nossa comunidade informal de amigos e colegas que podem ser confiáveis para ajudar a melhor atuar na complexidade do mundo (Vacik, Torresan, Hujala, Khadka, \& Reynolds, 2013).

Uma das mudanças mais importantes é observada através da alteração do foco que antes era pela mão de obra - e uma organização de produção Taylorista, e que hoje é centralizada no cérebro de obra (Vygotsky, 2007). APO (2009) esclarece que, se o conhecimento é o principal recurso produtivo na atualidade, sua gestão é totalmente prioritária nas empresas de diversas dimensões. Desta forma, torna-se um desafio nas várias organizações, pois a gestão adequada do conhecimento como elemento agregador de valor, deve estar alinhada e direcionada com a própria operação, para um uso ótimo dos recursos.

Outros pensamentos indicam que a GC muda o foco do processo de prática. A GC deve ser usada para comunicação e a colaboração para aprimorar a forma como as pessoas fazem a sua prática (o seu trabalho dentro de um processo global) (Ghani, 2009; Heisig, 2009; Porter \& Krammer, 2011; Senge, 2000). A gestão desses ativos, segundo Teixeira e Valentim (2012), compreende tanto nos níveis estratégicos e operacionais, e, pelos seus resultados reforçam a importância da socialização das estratégias organizacionais.

Acadêmicos no campo da GC normalmente definem conhecimento como um derivado de informação, que é proveniente a partir dos dados. Enquanto informações são dados organizados com um significado (North \& Rivas, 2010), o conhecimento é informação contextualizada que 
possibilita agir (Drucker, 1999). A ideia central é que os esforços de GC devam trabalhar para criar, codificar, compartilhar e armazenar conhecimentos que permitam a criação de valor e auxiliar o processo de tomada de decisão na organização.

Gestão do Conhecimento (GC) é a construção de inteligência organizacional, que permite às pessoas melhorarem o uso do conhecimento. Trata-se de usar as idéias e experiência dos funcionários, clientes e fornecedores para melhorar o desempenho da organização. Com base no que funciona bem leva a uma melhor prática, estratégia e política (Leask, Lee, Milner, Norton, \& Rathod, 2008).

Isto sugere que a proliferação de conhecimento pode exigir tempo e proficiência do usuário para selecionar as informações disponíveis. Como resultado, há uma crescente necessidade de estratégias de troca de conhecimentos que compartilhem rápido, com confiabilidade e fácil acessibilidade aos domínios específicos (Mairs, McNeil, McLeod, Prorok, \& Stolee, 2013).

Os métodos e técnicas de GC adaptam o entendimento das abordagens dos métodos e técnicas usados frequentemente na GC nas organizações. Em particular, os métodos fornecem passos essenciais para mapear a GC como instrumento para identificar, criar, disseminar, estocar e aplicar conhecimento nas organizações, no sentido de gerar sustentabilidade como aparece em APO (2009); Heisig (2009); Comité Europeo de Normalización [CEN] (2004). De certo modo, os métodos são destinados aos usuários com compreensão, em profundidade, nos fundamentos sobre GC.

As organizações precisam saber: quais são os seus ativos de conhecimento; quais são os seus processos relacionados de conhecimento; como gerenciar e fazer uso desses ativos e processos para obter o máximo de retorno (Mackintosh; Kingston; Filby, 1999).

Mairs et al. (2013) consideram as tecnologias da informação e comunicação um meio pragmático e pontual para transferir seletivamente pesquisa valiosa para os decisores políticos, profissionais, o público e outras partes interessadas. Os autores realizaram uma pesquisa e revisão sistemática para avaliar o uso de estratégias online para a transferência de conhecimento, entre 2003 e 2012. Dez dos artigos revisados (22\%) utilizaram um sistema de GC como a sua única estratégia para a tradução do conhecimento, enquanto seis (13\%) dos artigos utilizaram um sistema de gestão do conhecimento em combinação com outra estratégia. Todas as estratégias de gestão do conhecimento incluiam um componente interativo que permitia aos usuários enviar comentários ou compartilhar o conhecimento entre outros membros do público-alvo. Dentre as práticas, foram citadas comunidades de prática virtuais, fóruns de discussão, ferramentas de conferência.

Choudhary, Harding, Camarinha-Matos, Koh, \& Tiwari, (2013) trabalharam com 17 artigos para abordar e exemplificar uma variedade de aspectos relacionados ao gerenciamento de conhecimento, para suportar vários tipos de redes de colaboração. Alguns destes trabalhos relacionaram com mecanismos de integração do conhecimento em cadeias produtivas de colaboração, de forma a incentivar o compartilhamento e enriquecimento do conhecimento. Em outros casos, os modelos de suporte à decisão nas empresas, bem como os diferentes processos de GC (ou seja, a aquisição de conhecimento e difusão) afetaram o desempenho econômico dos fabricantes em intercâmbios colaborativos com seus fornecedores. As contribuições de relações interorganizacionais e as práticas de gestão do conhecimento como preditores de adoção de comércio colaborativo (c-commerce), tambem foram citadas. O papel da Internet se sobressai como uma tecnologia capacitadora para as redes virtuais de colaboração entre Pequenas e Médias Empresas (PMEs).

Leask et al. (2008) têm a visão que os métodos e técnicas devem auxiliar todos os setores para facilmente encontrar, usar, criar, gerir e disseminar conhecimento. Para responder: O que é? Por que e como utilizar? Deverão atuar na conexão de pessoas, grupos e organizações para gerenciar o fluxo informacional e inteligência organizacional.

Em decorrência dos seus objetivos (caracterizar e analisar os métodos e técnicas de GC na prática), a presente pesquisa utiliza as práticas de GC apresentadas pela APO (2010) como elementos-chave para suportar os processos de GC. Tais práticas permitem utilizar as dimensões aceleradoras, atingir os objetivos de GC nas dimensões de aprendizagem e inovação e de resultados de desempenho. 
A competência de gerir o conhecimento organizacional diz respeito à forma de uma organização olhar para o seu conhecimento e aprendizagem de maneira estratégica. Os métodos e técnicas de GC fornecem diferentes aplicações no capital humano, estrutural e relacional - ou seja, no capital intelectual - que podem ser usados para planejar, monitorar e avaliar o conhecimento e iniciativas de aprendizagem (APO, 2009; Ramalingam, 2006; Sveiby, 1998).

\section{Capital Intelectual e Business Inteligence}

Nesta nova era de organizações que utilizam o conhecimento como elemento agregador de valor, o Capital Intelectual (Cl) se destaca como um fator determinante de vantagem competitiva (Bontis, 2001; Kaplan \& Norton, 1996; Sveiby, 1997). Diante disto, o Capital Intelectual pode ser entendido como a soma dos ativos imateriais da organização, sendo este criado a partir do intercâmbio de diferentes dimensões, como capital humano, estrutural e relacional (Stewart, 1997; Edvinson \& Malone, 1997; Roos \& Roos, 1997), conforme mostra a Figura 01.

O capital humano refere-se às pessoas como fonte de riqueza das organizações, é composto pelas capacidades individuais, conhecimentos, habilidades e experiências, tornando-se fontes de inovação e renovação estratégica (Rodrigues, Dorrego, Fernández, \& Fernández, 2009; Wiig, 1997).

Malavski, de Lima, \& da Costa (2010) definem o capital estrutural como sendo o conhecimento apropriado pela empresa, este engloba processos organizacionais, softwares, procedimentos, sistemas, banco de dados, etc. Neste sentido, o capital estrutural engloba o capital humano e possibilita que a organização a utilize depois, aperfeiçoando indivíduos e a própria organização (Massingham, 2008).

Já a soma de todos os recursos associados às relações externas da empresa, deve ser definida como o capital relacional da organização (Malavski et al., 2010). Este trata do conhecimento que se incorpora à organização como consequência do valor derivado do número e da qualidade das relações com diferentes agentes de mercado e a sociedade em geral (Bueno, Real, Fernández, Longo, Merino, Murcia, \& Salmador, 2011).

Na Figura 1, são apresentadas as dimensões do Cl (Capital Humano, Estrutural e Relacional), com base na categorização de Sveiby (1997), bem como uma nova visão para guiar a formulação de estratégias baseada em conhecimento: as diferentes formas de transferência entre os recursos intangíveis oriundos das competências individuais, estrutura interna e externa (Sveiby, 2001).

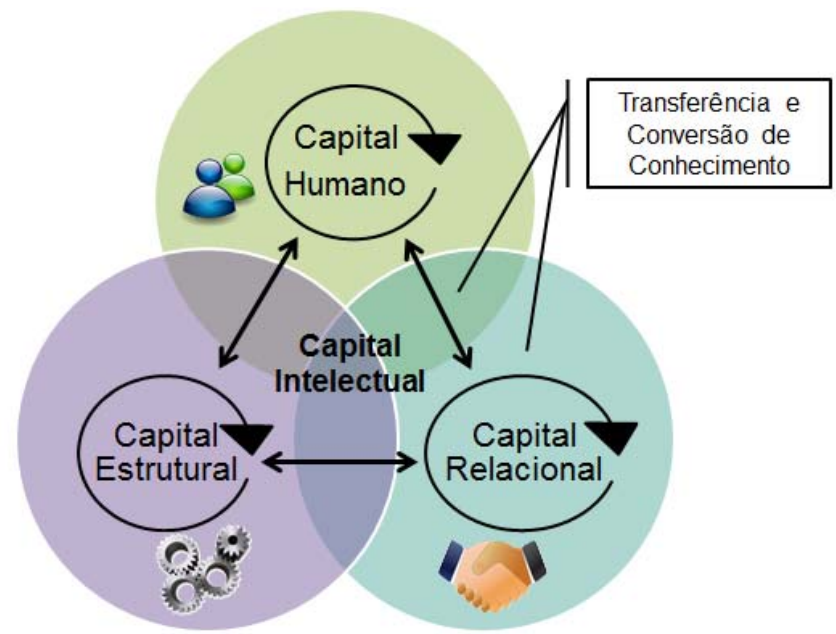

Figura 1. Dimensões do Capital Intelectual e Transferência e Conversão de Conhecimento Fonte: Adaptado de Sveiby $(1997 ; 2001)$

Neste sentido, o Capital Intelectual é concebido como a combinação da "criação de valor" entre o Capital Humano de uma empresa (habilidades, experiência, competência e capacidade de inovação pessoal), o Capital Estrutural (processos e sistemas organizacionais, software, bases de dados e processos de negócio), e Capital Relacional (todos os recursos ligados aos relacionamentos 
externos da empresa com os stakeholders, tais como, clientes, credores, investidores, fornecedores etc.) (MERITUM, 2002).

A relação entre $\mathrm{Cl}$ e $\mathrm{GC}$ é importante, porque os relatórios de $\mathrm{Cl}$ informam sobre as atividades que o gerenciamento inicia e apoia em nome da GC (Bukh, Larsen, \& Mouritsen, 2001).

A importância do pensamento orientado a dados não é nova. Muitos executivos estão familiarizados com o conceito. A ascensão das empresas orientadas a dados, do Facebook ao Walmart, mostra quão poderosa a abordagem pode ser na prática. Desta forma, torna-se necessário compreender os benefícios dos métodos e técnicas de GC para a adoção de uma cultura orientada a dados dentro de uma organização.

Em outubro de 1958 a IBM - Journal of Research and Development - apresentava o artigo de Hans Peter Lunh:

\footnotetext{
Um sistema automático está sendo desenvolvido para divulgar informações para as várias seções de qualquer organização científica, industrial ou governamental. Este sistema de inteligência vai utilizar máquinas de processamento de dados para a auto-abstração e auto-codificação de documentos e para a criação de perfis de interesse para cada um dos "pontos de ação" de uma organização. (Lunh, 1958, p. 314)
}

Watson e Wixom (2007) fizeram um relato sobre o estado da arte do Bl. Comentam que no início da década de 90 do século passado Howard Dresser, então analista da Gartner Group, cunhou o termo Business Intelligence. O Bl é agora amplamente usado, especialmente no mundo prático, para descrever aplicações analíticas, sendo atualmente a prioridade número um de muitos CEO's (Chief Executive Officer). Em uma pesquisa com 1.400 CEOs, o Gartner Group constatou que os projetos de $\mathrm{Bl}$ foram a prioridade número um de tecnologia para 2007, o BI "tornou-se uma iniciativa estratégica e é agora reconhecido por CEO's e líderes empresariais como instrumental em conduzir a eficácia do negócio e inovação".

Sistemas de $\mathrm{Bl}$ combinam dados operacionais com ferramentas analíticas para apresentar informação complexa e competitiva. Desta forma, o BI é uma coleção de tecnologias de apoio à decisão para a empresa destinada a permitir que os trabalhadores do conhecimento, tais como executivos, gerentes e analistas para tomar decisões melhores e mais rápidas. BI é usado para entender os recursos disponíveis na empresa, o estado da arte, tendências e direções futuras nos mercados, as tecnologias e do ambiente regulatório no qual a empresa concorre; as ações dos concorrentes e as implicações das mesmas (Negash, 2004; Chaudhuri, Dayal, \& Narasayya, 2011).

Este crescimento foi impulsionado pelo declínio do custo de adquirir e armazenar grandes quantidades de dados provenientes de fontes como transações de clientes no setor bancário, de varejo, bem como em e-business, identificação por rádio frequência (RFID) para controle de estoque, e-mail, logs de consulta para sites da Web, blogs e análises de produtos (Chaudhuri, Dayal, \& Narasayya, 2011) Um framework, desenvolvido por Watson e Wixom (2007) pode ser observado na Figura 2. 


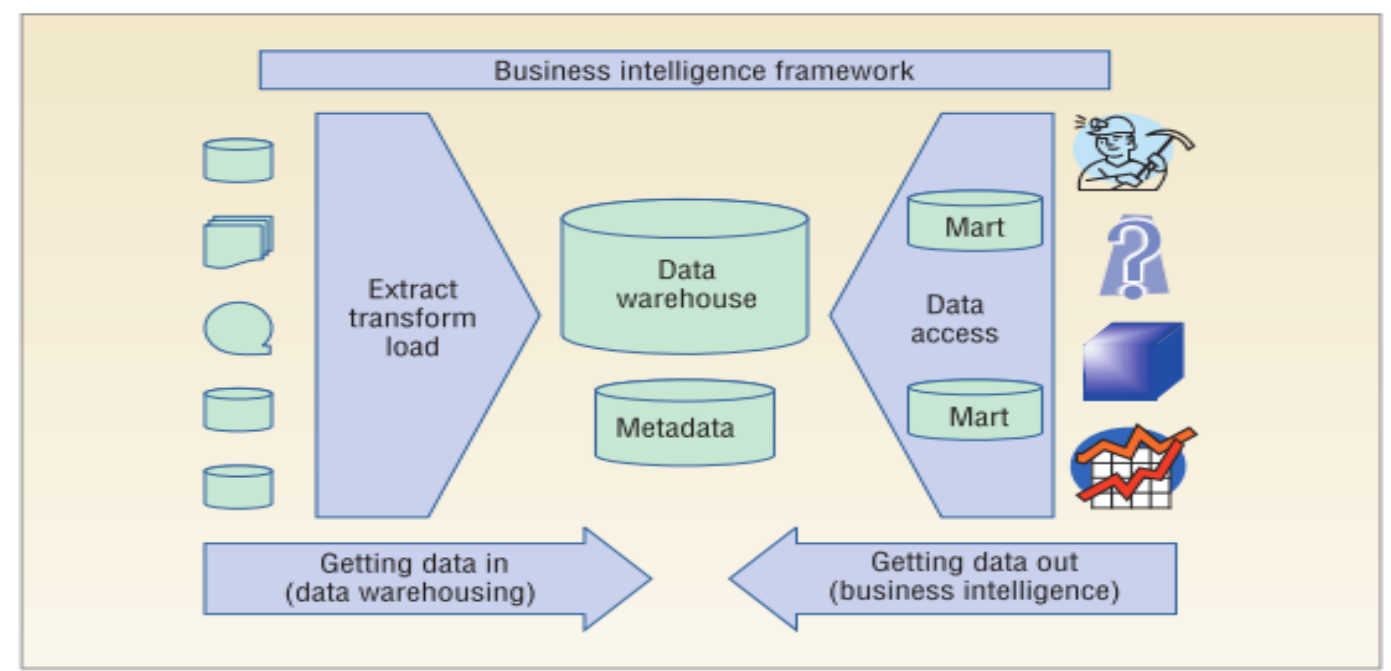

Figura 2. Quadro referencial de Business Intellgence. O BI inclui duas atividades primárias, obter dados internos e formação/liberação de dados.

Nota: Baseado em Watson e Wixom (2007), optou-se por preservar a figura na língua inglesa a fim de evitar distorções das expressões e traduções. O framework apresenta o modelo de desevolvimento no Business Intelligence mostra os elementos de dados e sua transformação. Por um lado encontram-se a extração, transformação e acúmulo dos dados, por outro o seu acesso, por meio de ferramantas inteligentes que possibilitam uma análise mais detalhada.

Para Chen, Chiang e Storey (2012), a evolução da BI está no modelo 3.0, cujos pontos chave ocorrem através de celulares e conteúdo baseado em sensores, sendo fornecidas: análise consciente de localização, análise centrada na pessoa, análise pertinente ao contexto e visualização nos celulares e Interação Humano-Computador (IHC).

O relatório de outubro de 2012 da revista The Economist, na unidade de Inteligência Econômica, relata a importância de fomentar a cultura conduzida a dados, apresentando os desafios e a forma como as empresas precisam fazer para atingi-los. Apresenta ainda, informações comparativas de 533 executivos sêniores de empresas do mundo todo, onde aquelas com uma maior solidez financeira são referências na utilização dos dados de sua organização. E o êxito na promoção de uma cultura orientada a dados se dá $49 \%$ em função da "Orientação top-down e/ou ordem de executivos", seguido por $48 \%$ pela "promoção de práticas de dados compartilhados". A Revista Forbes (2014) cita as dez principais tendências para BI para o ano de 2014, conforme mostra a Figura 3.

A dez tendências elencadas, mostram a importância da BI como pode ser visto e aplicado nas organizações contribuindo para o seu desenvolvimento e competitividade. O que se destaca na análise da pesquisa da Forbes (2014) é a prática de gestão do conhecimento, storytelling, apontada como uma importante ferramenta de disseminação do conhecimento no contexto do BI e no tratamento de big data em organizações intensivas em conhecimento. 


\begin{tabular}{|c|c|c|}
\hline 1 & 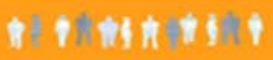 & Fim dos cientistas de dados \\
\hline 2 & & $\begin{array}{l}\text { Nuvem de inteligência de negócios será o influxo } \\
\text { principal }\end{array}$ \\
\hline 3 & 2 & Big Data finalmente irá decolar \\
\hline 4 & 2) & Agilidade da BI ampliará a liderança das empresas \\
\hline 5 & & As análises serão preditivas \\
\hline 6 & Ex & $\begin{array}{l}\text { As empresas que tiverem } \mathrm{BI} \text { incorporadas começam } \\
\text { a emergir }\end{array}$ \\
\hline 7 & & $\begin{array}{l}\text { Storytelling torna-se uma prioridade na } \\
\text { disseminação do conhecimento }\end{array}$ \\
\hline 8 & & $\begin{array}{l}\text { Para as organizações de ponta, Mobile Business } \\
\text { Intelligence, não será uma experiência ocasional }\end{array}$ \\
\hline 9 & & $\begin{array}{l}\text { Organizações começam a analisar os dados sociais } \\
\text { em série }\end{array}$ \\
\hline 10 & & $\begin{array}{l}\text { Organizações irão procurar explorar como usar os } \\
\text { dados não estruturados }\end{array}$ \\
\hline
\end{tabular}

Figura 3. Dez tendências para o Business Intelligence segundo a Forbes (2014)

Nota: Adaptado de Forbes (2014), a imagem construída a partir dos elementos apontados na pesquisa, mostram as características e vantagens do BI no campo organizacional, destacando a agilidade, predição e todo seu desenvolvimento por meio de ações e práticas de GC, com destaque para storytelling.

\section{Procedimentos Metodológicos}

Os objetivos do artigo foram avaliar e caracterizar os métodos e técnicas de GC usados por organizações intensivas em conhecimento e; relacionar esses procedimentos as dimensões do Capital Intelectual (capital humano, estrutural e relacional). Complementarmente, a pesquisa objetivou recomendar métodos e técnicas essenciais de GC alinhados às tendências do Business Inteligence.

A amostra incluiu 20 empresas, que correspondeu a $25 \%$ dos participantes ativos de uma associação de classe. A amostra continha empresas do setor de alimentos, saúde, metal mecânica, têxtil e serviços.

Em decorrência do seu primeiro objetivo específico (caracterizar e analisar os métodos e técnicas de GC na prática), a presente pesquisa utilizou as práticas de GC apresentadas pela APO (2010) como elementos-chave para suportar os processos de GC. Tais práticas permitem utilizar as dimensões aceleradoras, atingir os objetivos de GC nas dimensões de aprendizagem e inovação e de resultados de desempenho.

Preliminarmente, foram realizados dois encontros presenciais, durante as reuniões ordinárias de núcleos setoriais dessa entidade de classe, com a finalidade de explicar os objetivos da pesquisa e o método aplicado à pesquisa. Posteriormente, os questionários foram enviados por correio eletrônico pela coordenação da associação, no período entre junho e agosto de 2014. Os questionários foram adaptados com base no modelo da APO (2010) e foram respondidos pelos representantes de cada empresa na entidade de classe estudada. O questionário era composto por 55 perguntas distribuídas da seguinte forma: quatro questões ligadas as atividades para conexão entre pessoas, dez questões relacionadas à melhoria organizacional, e para os mecanismos de coordenação (seis perguntas para controle e coordenação social (externo a empresa), cinco questões ligadas a sistemas de controle e coordenação social (interno a empresa), três itens vinculados as unidades e papéis de integração, sete questões relativas aos sistemas de controle e planejamento, cinco interrogações referentes aos sistemas de incentivo da organização e cinco relativas aos sistemas de informação). Em cada questão foram listadas os diferentes métodos e técnicas de GC, com um breve comentário sobre seu funcionamento, procedimento e justificativa. 
Os respondentes avaliaram os métodos e técnicas utilizados, em uma escala de 0 a 4 , para a situação atual e situação desejada, conforma apresenta o Quadro 1.

Quadro 1. Escala de avaliação e situação aplicadas ao questionário

Situação Atual (como realmente é) Situação desejada (como tem que ser)

\begin{tabular}{llcl}
\hline $\mathbf{0}$ & Não é utilizada em nada & 0 & Não é utilizada em nada \\
$\mathbf{1}$ & Tem pouco alcance & 1 & Tem pouca aplicação \\
$\mathbf{2}$ & Certo alcance & 2 & Certa aplicação \\
$\mathbf{3}$ & Um alcance grande & 3 & Uma aplicação grande \\
$\mathbf{4}$ & Um alcance muito grande & 4 & Uma aplicação muito grande \\
\hline
\end{tabular}

Nota: Baseado na classificação da APO (2010) mostra como a escala que classifica as técnicas e ferramentas de gestão do conhecimento no que tange a sua situação atual até a situação desejada.

Os respondentes após realizar a escala de avaliação comentavam, em questão aberta, como e porquê ocorre o referido método e técnica de GC. Nas situações de desconhecimento da ferramenta ou técnica, o respondente deveria colocar índice zero para situação atual e indicar o fato. Neste sentido, foram avaliados os dados desta categorização como forma de caracterizar o enquadramento das técnicas e ferramentas diagnosticadas, conforme mostram os resultados a seguir.

\section{Apresentação e Análise dos Resultados}

As atividades relacionadas às conexões de pessoas 'ajuda a semelhantes' foram identificadas como a técnica mais utilizada e referendada como sendo de "uma aplicação muito grande". A segunda ferramenta mais aplicada foi 'comunidade prática'. Este resultado é justificado por Mairs et al. (2013) e que correlaciona a confiança mútua entre os colaboradores e a especificidade do conhecimento dos indivíduos e do grupo. A importância dada a estas duas técnicas é referendada pelo maior coeficiente como uma situação desejada a sua aplicação, contudo as aplicações desses métodos foram relacionadas a questões específicas e indicaram como uma prática rotineira; 'Comunidade prática' diferiu em valores médios do 'café do conhecimento'. No entanto, 'café do conhecimento' e 'feira do conhecimento' são avaliadas como técnicas de aplicação de "certo alcance" e com o maior fator de desconhecimento entre os métodos e técnicas. As técnicas preferenciais quanto ao alcance - aplicação muito grande - foram 'ajuda a semelhante' e 'comunidade de prática'. As distribuições dos valores podem ser observadas no Gráfico 1.

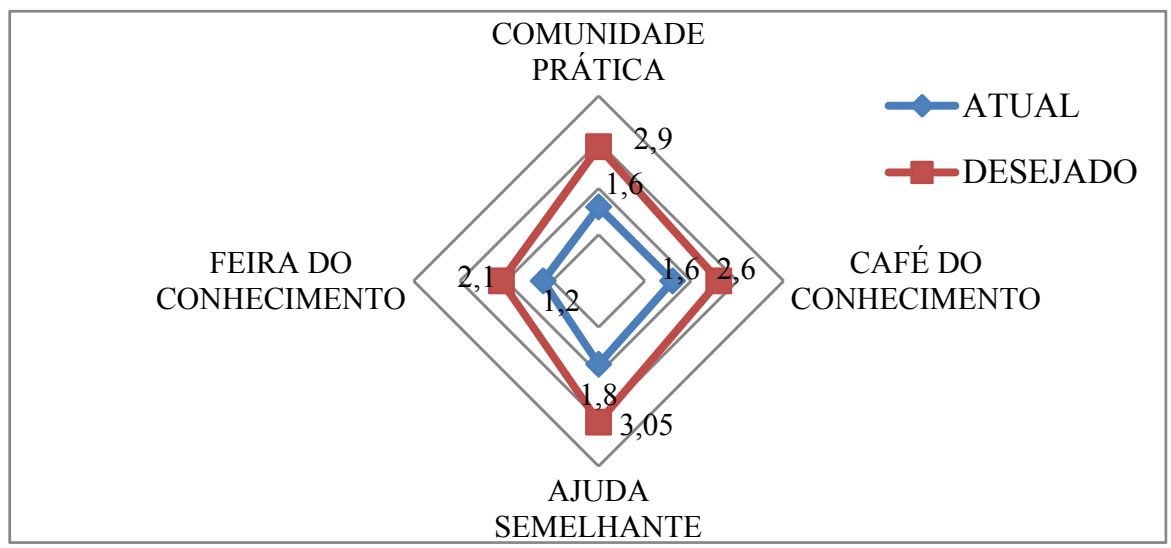

Gráfico 1. Atividade para Conexão de Pessoas em 20 Organizações Intensivas em Conhecimento - Julho (2014)

Nota: Elaborado pelos autores (2015) 
Os índices vinculados aos métodos e técnicas relacionados à melhoria organizacional estão presentes no Gráfico 2. Foram avaliados como efetivas as suas realizações, os métodos de 'reuniões informais' e 'grupos para solução', contudo foram caracterizadas como "certo alcance" para a situação atual de desempenho da técnica nas organizações inqueridas. 'Comunidade prática' foi percebida pelos entrevistados como o método de pior situação atual, bem como ser de "pouco alcance" para situações futuras desejadas. Salienta-se que sete respondentes relacionaram 'grupos para solução de problemas' como uma ocorrência informal para a solução de problemas, o que demonstrou uma composição com "Comunidade de Prática'.

O "alto alcance" indicado para 'reuniões informais' e 'grupos de solução' para as práticas de melhoria organizacional pode estar relacionado à contribuição que estes métodos proporcionam ao desenvolvimento das capacidades dos colaborados, de forma pessoa a pessoa, favorecendo a gestão do capital humano, ou seja, criação de rede de colaboradores, fato anteriormente referendado por Chaudhuri; Dayal; Narasayya (2011); Teixeira; Valentim (2012); Ghani (2009); CEN (2004); Ramalingam (2006); Chen; Chiang; Storey (2012). Conforme mostra o Gráfico 2.

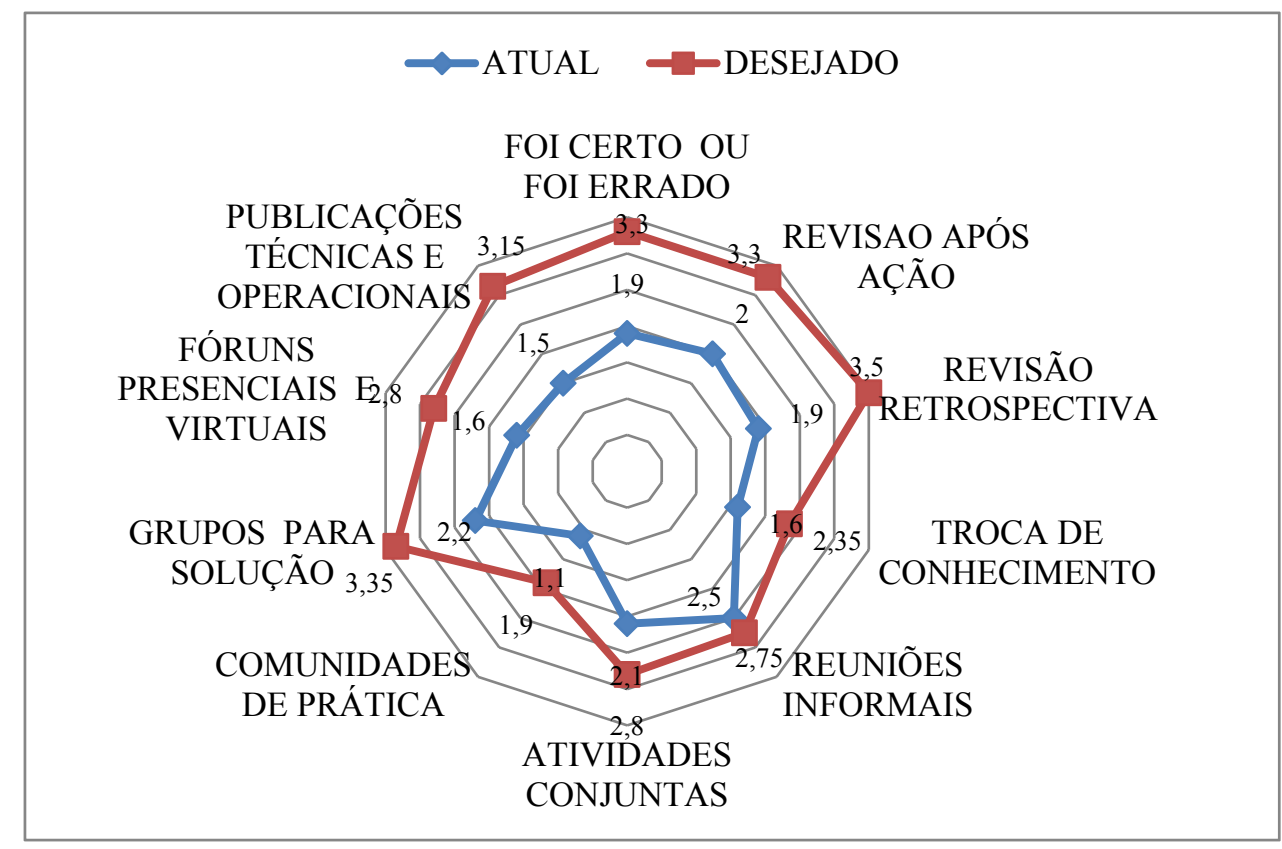

Gráfico 2. Atividade para melhoria organizacional em 20 Organizações Intensivas em Conhecimento Julho (2014)

Nota: Elaborado pelos autores (2015)

Os métodos de maior importância sendo considerados como "uma aplicação muito grande" (escore superior a três) foram em ordem decrescente de importância 'revisão pós-ação', com 3.5 pontos; seguido por 'grupo para solução' com 3.35 pontos; 'foi certo ou foi errado' e 'revisão retrospectiva', que obtiveram média de 3.3 pontos e 3.15 pontos para 'publicações técnicas e operacionais'. Explicita-se, pelos resultados, uma valoração pela identificação das consequências das rotinas aplicadas, ou seja, a gestão do conhecimento tem influência direta nos processos, ou seja, na prática, como corroboram Ghani (2009); Heisig (2009); Porter, Krammer (2011); Senge (2000); Teixeira, Valentim (2012). As técnicas que obtiveram o menor intervalo entre a situação atual e a desejada foram 'reuniões informais' ( 0.25 - 2.5 atual e 2.75 desejada) e 'atividades conjuntas' (0.7 - 2.1 atual e 2.8 desejada). 'Revisão retrospectiva' obteve a maior amplitude entre o praticado e o desejado, na ordem, 1.9 e 3.5.

Para mecanismos ligados ao controle e coordenação social tiveram destaque os métodos de 'conselheiro de campo', 'mestre aprendiz' e 'espaço compartilhado' pela alta frequência de desconhecimento e, consequentemente, sua aplicação. Vale ressaltar que 'mestre aprendiz' teve a maior amplitude entre o realizado e o desejado, o indica um interesse na aplicação deste método. 
'Observação real' teve valores semelhantes entre sua aplicação e situação desejada, o que permite afirmar que há bom uso do método. Concomitantemente, foi a terceiro método menos conhecido pelos entrevistados (75\%). Destacaram-se positivamente 'visitas técnicas entre empresas' e 'coaching'. Essas técnicas têm grande relação com capacitações através dos capitais humano e relacional. E ambas demostram que há uma lacuna para o aprimoramento do seu uso nas organizações avaliadas. A aplicação efetiva dessas técnicas permite suprir as recomendações de Leask et al. (2008), para a melhoria e desenvolvimento no auxílio a acessar o correto conhecimento e no momento correto, como mostra o Gráfico 3.

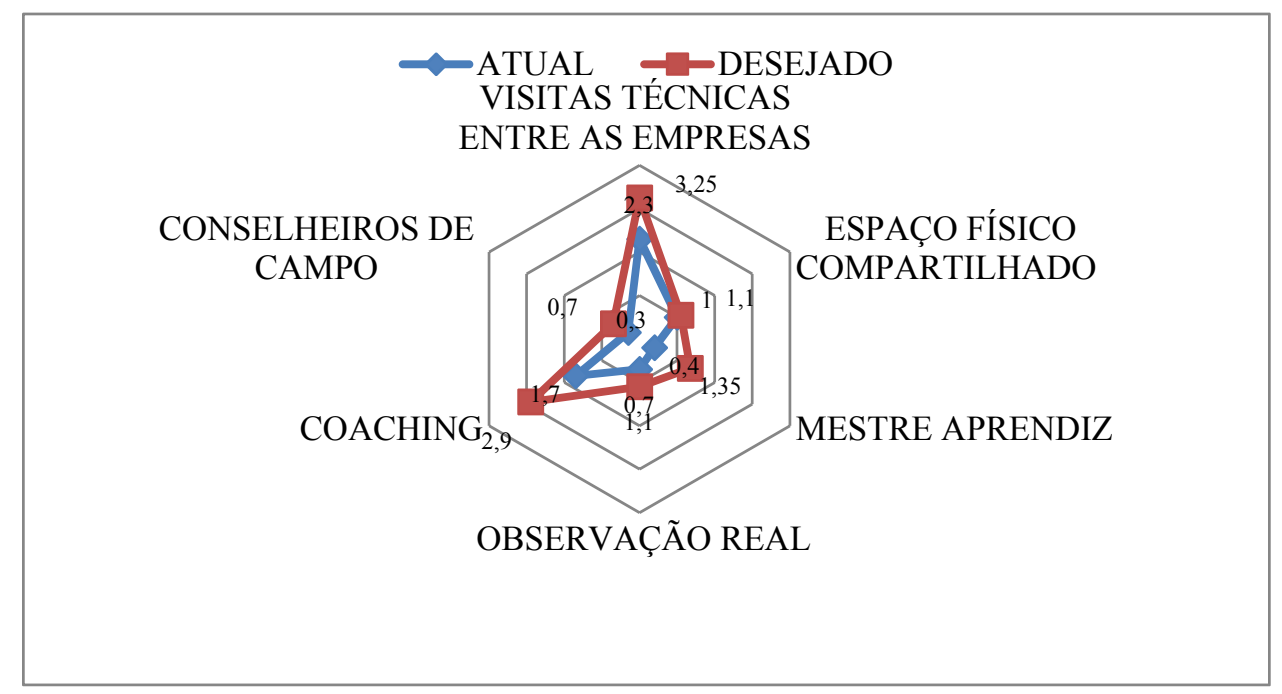

Gráfico 3. Atividade para Mecanismos de Coordenação Social para controle e coordenação social em 20 Organizações Intensivas em Conhecimento - Julho (2014)

Nota: Elaborado pelos autores (2015)

Mecanismos de coordenação para unidade e papéis de integração, 'inteligência competitiva' obteve frequência de desconhecimento por $80 \%$ dos participantes. A técnica mais aplicada foi 'elaboração de manuais para replicação de práticas', assim como foi a mais importante, considerada no intervalo de "uma grande aplicação" nas organizações avaliadas. Em seguida, foram o 'benchmarking interno e externo' e 'rotinas bem-sucedidas' quanto ao uso e, uma inversão de ordem nas posições para situações desejadas. Fica evidente que as três técnicas mais aplicadas têm influência direta no capital estrutural das empresas participantes. São exemplos a 'explicitação do conhecimento em manuais', o 'benchmarking interno e externo', que permitem a incorporação de novos ou processos produtivos melhorados, e a disseminação de rotinas bem-sucedidas. Esses métodos e técnicas permitem, se coordenadas pela GC, mapear para identificar, criar, disseminar, estocar e aplicar conhecimento nas organizações, no sentido de gerar sustentabilidade, como recomendam Drucker (1999); APO (2009), Heisig (2009); CEN (2004); Leask et al. (2008). Assim, é possível afirmar que as empresas têm uma predisposição e capacidade importante de aprendizagem.

Mecanismos de coordenação para sistemas de informação 'espaços eletrônicos' (portais, emails, chats, intranet e extranet) foi o mais utilizado e mais valorado para aplicação na empresa $90 \%$ de frequência na escala de situação desejada de "uma aplicação muito grande". "Gestão eletrônica de documentos' foi elencada como a segunda no atual alcance da ferramenta dentro da organização, bem como na aplicação desejada. Esses mecanismos de coordenação estão aliados aos pensamentos descritos por Mairs et al. (2013); North; Rivas (2010); Mackintosh et al. (1999), que permite acessar com facilidade, rapidez e confiabilidade os dados organizados com significado. No entanto, esses dados e informações deverão estar corretamente armazenados de modo que realize as funções descritas por Mairs et al. (2013). 
'Base de dados compartilhados online' e 'espaço eletrônico: e-learning' foram de baixo uso, posicionaram-se na primeira e terceira posição quanto à aplicação e com alta frequência para a falta de informação sobre a técnica, respectivamente. 'Gestão de conteúdo' também foi elencada no grupo dos métodos com grau de desconhecimento. No entanto, apresentou escala máxima para situação desejada de aplicabilidade para as organizações que aplicam a ferramenta.

A gestão eletrônica dos documentos e os espaços virtuais colaborativos (e-mail, internet, entre outros) representam práticas de GC que aprimoram diretamente o Capital Estrutural e relacional das organizações, por intermédio de uma melhor explicitação e compartilhamento do conhecimento.

\section{Recomendações}

Gerenciar adequadamente o conhecimento tem uma influência direta nos resultados da organização, nas dimensões de desempenho e aprendizagem (APO, 2009). A Confederação Nacional da Indústria [CNI] (2010) recomenda para a Mobilização Empresarial pela Inovação (MEI), um plano de ação, específico e detalhado para cada setor que deverá ser construído de forma coletiva e participativa, envolvendo os diversos atores e grupos envolvidos nos processos das organizações.

O gerenciamento é colocado em prática através de métodos e técnicas de GC que atuam de forma combinada. O estudo proposto apresenta recomendações que indicam caminhos fundamentados nas práticas de GC com melhor desempenho na sua aplicação bem como sua avaliação de aplicabilidade por intermédio dos escores indicados pelos entrevistados após serem quantificados e analisados. A recomendação tem por base o uso de múltiplos métodos e técnicas, referendado pelo trabalho de Mairs et al. (2013) tendo em vista a transferência e conversão de conhecimento para avanços no $\mathrm{Cl}$ (Sveiby, 1997) com objetivo da aplicação estratégica de Business Intelligence proposta pela (Forbes, 2014). Na Figura 4, podem ser observadas as recomendações feitas referentes às práticas e ferramentas que auxiliam o desenvolvimento de uma cultura orientada ao Capital Intelectual e Business Inteligence.

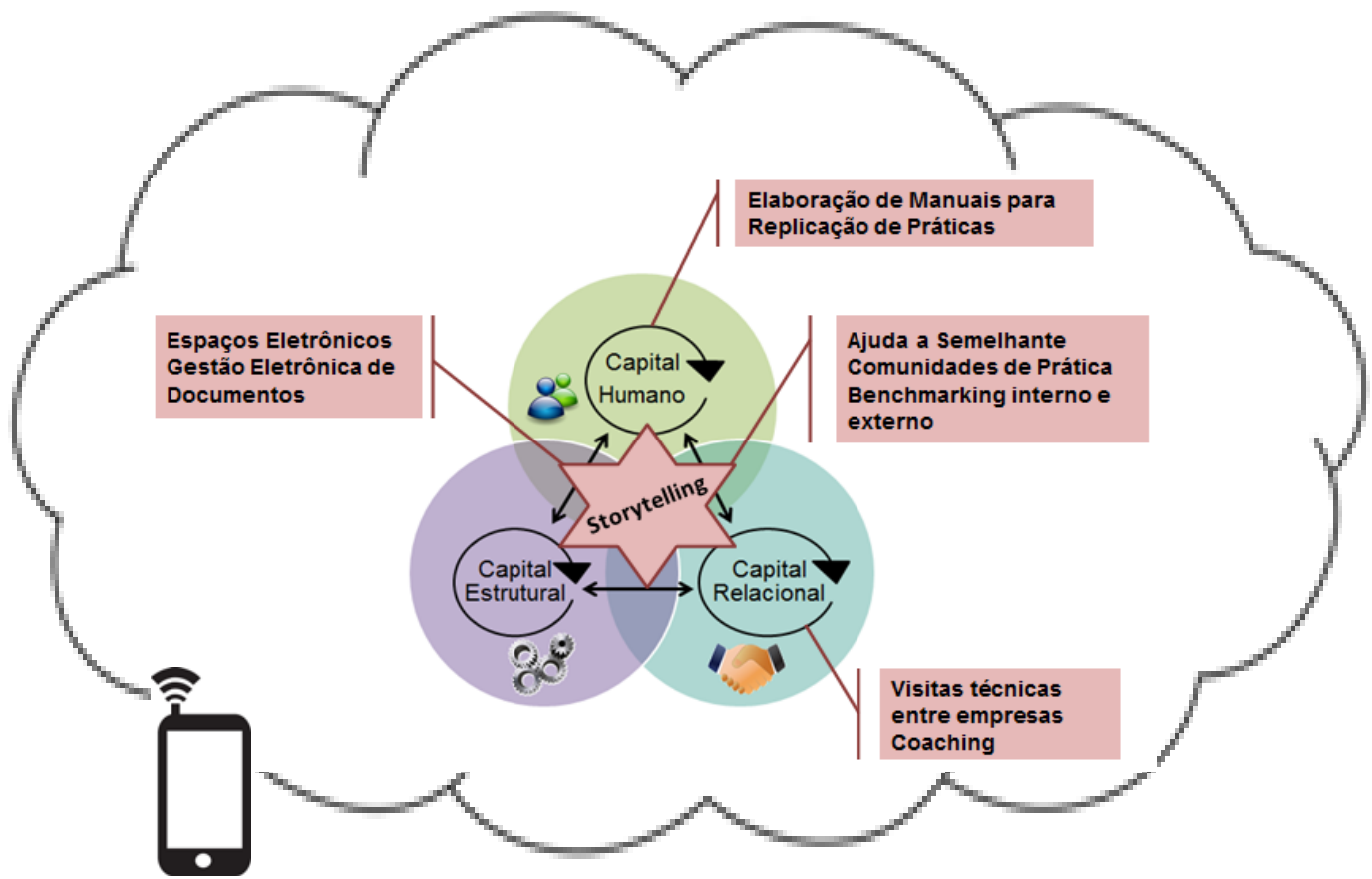

Figura 4. Recomendações feitas para desenvolvimento do Business Intelligence com base no levantamento dos Métodos e Técnicas de GC alinhados às dimensões do Capital Intelectual Nota: Adaptado de Sveiby (1997; 2001), foram relacionadas as ferramentas e técnicas de GC com as dimensões do capital intelectual (capital humano, estrutural e relacional) conforme dados apresentados na pesquisa realizada relacionado ao Business Inteligence. 
Para a GC, referente ao Capital Humano foram relacionadas as ferramentas para a conexão de pessoas a 'ajuda semelhante' e 'Comunidade Prática', que terão aplicabilidade também na transferência e conversão do conhecimento, juntamente ao Capital Relacional, os fornecedores e concorrentes. Aliam-se nesse processo entre $\mathrm{CH}$ e $\mathrm{CR}$ as técnicas de 'Comunidade Prática' e 'Bechmarking interno e externo'. No compartilhamento de conhecimento no CR são recomendadas as 'visitas técnicas entre empresas' e 'coaching'. 'Espaços eletrônicos' e 'Gestão eletrônica de documentos' possibilitarão com mecanismos de controle para sistemas de informação transferência e conversão de conhecimento entre o CE e CH. A técnica referencia 'Storytelling' e possibilita atuar nos seis pontos de transferência, estocagem e conversão de conhecimento para todas as dimensões do $\mathrm{Cl}$ e pela recomendação da (Forbes, 2014).

\section{Conclusões}

Os métodos e técnicas de GC sendo corretamente aplicados poderão permitir que a "ciência dos dados" se mova do especialista para o compartilhamento com o homem comum. A familiaridade com a análise de dados torna-se parte do conjunto de habilidades e capacidades dos usuários comuns do negócio, sem a especialidade de "analista" no seu currículo.

O uso dos 'espaços eletrônicos' e 'gestão eletrônica de documentos' permitirá as organizações que desejam se agilizar e ter rapidez em suas análises impulsionar a adoção de "Business Intelligence Cloudbased". Principalmente com a exigência de cenários colaborativos entre todos os integrantes da cadeia produtiva. Essas técnicas permitirão "autoatendimento analítico" (Agile Business Intelligence) que devidamente criada e armazenada permitirá que empresas decidam rapidamente através da fexibilidade e usabilidade de seus dashboards (gráficos). Uma vez aplicados, os sistemas avançados e especializados, tornar-se-ão a corrente principal para as empresas que buscam o futuro, visão predictiva, em vez do olhar retroativo dos dados.

Os indivíduos, os grupos e as organizações em função dos inúmeros dados e infomações fornecidas pelos gráficos sem contexto tornam-se inúteis. A prática de "Storytelling" (narrativas) se torna uma forma ágil de comunicar idéias e insights usando esses artefatos. Assim esse método terá função central na Gestão do Conhecimento, com possiblidades de integrar todos os componentes do Capital Intelectual - Capital Humano, Capital Relacional e Capital Estrutural.

\section{Agradecimentos}

Este trabalho foi subsidiado por meio do órgão de fomento à pesquisa CAPES, por meio da disponibilização de bolsas para os pesquisadores.

\section{Referências}

APO (2009). Knowledge management: Case studies for small and medium enterprises. Tokyo: APO.

Bontis, N. (2001). Assessing knowledge assets: A review of the models used to measure intellectual capital. International Journal of Management Reviews, 3(1), 41-60.

Bontis, N. N. (2001). Thought leadership on intellectual capital. Journal of Intellectual Capital, 2(3), 183-191.

Bueno, E., Real, H. D., Fernández, P., Longo, M., Merino, C., Murcia, C., \& Salmador, M. (2011). Modelo intellectus: Medición y gestión del capital intelectual. Documentos Intellectus. Madrid: Universidad Autónoma de Madrid.

Bukh, P. N., Larsen, H. T., \& Mouritsen, J. (2001). Constructing intellectual capital statements. Scandinavian Journal of Management, 17(1), 87-108. 
Canibano, L. Garcia Ayuso, M. Sanchez, P. Chaminade, C. (Eds) (2002). Guidelines for managing and reporting on intangibles (Intellectual Capital Report). Madrid: Airtel-Vodafone Foundation.

Comité Europeo de Normalización - CEN. (2004). European guide to good practice in knowledge management: Part 1 - 5. Brussels: CEN Workshop Agreement.

Chaudhuri, S., Dayal, U., \& Narasayya, V. (2011). An overview of business intelligence technology. Communications of the ACM, 54(8), 88 - 98.

Chen, H., Chiang, R. H., \& Storey, V. C. (2012). Business intelligence and analytics: From big data to big impact. MIS Quarterly, 36(4), 1165-1188.

Choudhary, A. K., Harding, J., Camarinha-Matos, L. M., Koh, M. C., \& Tiwari, M. K. (2013). Knowledge management and supporting tools for collaborative networks. International Journal of Production Research, 51(7), 1953-1957.

CNI - Confederação Nacional da Indústria. (2010). Mobilização empresarial pela inovação: Manual de orientação de núcleos. Brasília: Confederação Nacional da Indústria.

Drucker, P. (1999). Management challenges for the twenty-first century. Oxon, UK: Routledge.

Edvinsson, L., \& Malone, M. S. (1997). Intellectual capital: Realizing your company's true value by finding its hidden brainpower. New York: Harper Business.

Forbes. (2013). Top 10 tend of business intelligence 2014. Recuperado de http://www.forbes.com/sites/peterhigh/2013/11/25/forrester-top-technology-trends-for2014-and-beyond/

Ghani, S. R. (2009). Knowledge management: Tools and techniques. DESIDOC Journal of Library \& Information Technology, 29(6), 33-38.

Heisig, P. (2009). Harmonisation of knowledge management: Comparing $160 \mathrm{KM}$ frameworks around the globe. Journal of knowledge management, 13(4), 4-31.

Kaplan, R., \& Norton, D. P. (1996). The balanced scorecard. Brighton, MA: Harvard Business Publishing.

Leask, M., Lee, C., Milner, T., Norton, M., \& Rathod, D. (2008). Knowledge management tools and techniques: Improvement and development agency for local government helping you access the right knowledge at the right time. London: IDeA.

Lunh, H. P. (1958). A business intelligence system. IBM Journal of Research and Development, 2(4), 314-319.

Mackintosh, A., Kingston, J., \& Filby, I. (1999). Knowledge management techniques: Teaching \& dissemination concepts. Edimburg: University of Edimburg.

Mairs, K., McNeil, H., McLeod, J., Prorok, J. C., \& Stolee, P. (2013). Online strategies to facilitate health-related knoeledge transfer: A systematic search and review. Health Information \& Libraries Journal, 30(4), 261 - 277.

Malavski, O. S., de Lima, E. P., \& da Costa, S. E. (2010). Modelo para a mensuração do capital intelectual: Uma abordagem fundamentada em recursos. Produção, 20(3), 439-454. 
Massingham, P. (2008). Measuring the impact of knowledge loss: More than ripples on a pond? Management Learning, 39(5), 541-560.

Negash, S. (2004). Business intelligence. Communications of the Association for Information Systems, $13,177-195$.

North, K., \& Rivas, R. (2010). Gestión del conocimiento: Una guía práctica hacia la empresa inteligente. Buenos Aires: LibrosEnRed.

Porter, M. E., \& Krammer, M. R. (2011). The big idea: Creating shared value - how to re-invent capitalism - and unleash a wave of innovation and growth. Recuperado de http://www.hks.harvard.edu/mrcbg/fellows/N_Lovegrove_Study_Group/Session_1/Michael_P ort

Ramalingam, B. (2006). Tools for knowledge and learning: A guide for development and humanitarian organisations. London: Research and Policy in Development Programme.

Rodrigues, H. M. da S. S.; Dorrego, P. F. F.; Fernández, C. M.; Fernández, J. (2009). La influencia del capital intelectual en la capacidad de inovación de las empresas del sector de automación de la eurorregión Galicia Norte de Portugal. Vigo: Universidad de Vigo.

Roos, G.; Roos, J. (1997). Measuring your company's intellectual performance. Long range planning, $30,413-426$,

Senge, P. M. (2000). The fifth discipline: The art \& practice of the learning organization. São Paulo: Best Seller.

Sveiby, K. E. (1997). The intangible assets monitor. Journal of Human Resource Costing \& Accounting, 2(1), 73-97.

Sveiby, K. E. (1998). A nova riqueza das organizações: Gerenciando e avaliando patrimônios de conhecimento. Rio de Janeiro: Editora Campus.

Teixeira, T. M., \& Valentim, M. L. (2012). Estratégias para disseminação do conhecimento organizacional: O papel da arquitetura da informação. Inf. Inf., 17(3), 165 - 180.

Vacik, H., Torresan, C., Hujala, T., Khadka, C., \& Reynolds, K. (2013). The role of knowledge management tools in supporting sustainable forest management. Forest Systems, 22(3), 442455.

Vygotsky, L. S. (2007). A formação social da mente. (7 ed.). São Paulo: Martins Fontes.

Watson, H. J., \& Wixom, B. H. (2007). The current state of business intelligence. Computer, 40(9), 96 99.

Wiig, K.M. (1997) Integrating intellectual capital and knowledge management. Long range planning, 30(3), 399- 405. 